\title{
Simultaneous measurement of liquid level and temperature employing a single uniform FBG
}

Xuewen Shu, Kate Sugden, Ian Bennion

Xuewen Shu, Kate Sugden, Ian Bennion, "Simultaneous measurement of liquid level and temperature employing a single uniform FBG," Proc. SPIE 7503, 20th International Conference on Optical Fibre Sensors, 75032 S (5 October 2009); doi: 10.1117/12.833528

SDIE Event: 20th International Conference on Optical Fibre Sensors, 2009, Edinburgh, United Kingdom 


\title{
Simultaneous measurement of liquid level and temperature employing a single uniform FBG
}

\author{
Xuewen Shu, Kate Sugden, Ian Bennion \\ Photonics Research Group, Aston University, Aston Triangle, Birmingham B4 7ET, United Kingdom \\ Tel: +4401212043549, email:X.Shu@aston.ac.uk
}

\begin{abstract}
We report a novel liquid level sensor based on a simple uniform fiber Bragg grating by monitoring both the short-wavelength-loss peaks and its Bragg resonance. The liquid level can be measured from the amplitude changes of the short-wavelength-loss peaks, while temperature can be measured from the wavelength shift of the Bragg resonance. Such a scheme has some advantages including robustness, simplicity, flexibility in choosing sensitivity, and simultaneous temperature measurement capability.
\end{abstract}

Keywords: Fiber optics sensors, Fiber Bragg gratings, liquid-level sensor

\section{Introduction}

Liquid level measurement and control is very important and highly demanded in many industrial applications such as fuel storage systems, chemical processing, water supplies and treatment plants. Various liquid level sensing techniques based on electrical, mechanical, ultrasonic, and optical methods have been developed [1-6]. Electrical liquid level sensors are widely used, but their applicability is limited if the liquid to be monitored is conductive, or if the environment is potentially explosive and erosive. Due to its inherent advantages such as dielectric and immunity to electromagnetic interfaces, optical fiber based technology is particularly attractive for application in these rigorous conditions. Recently, some fiber grating based level sensing schemes have been demonstrated, such as using long-period gratings (LPGs) [4], etched fiber Bragg gratings (FBGs) [5], and FBGs on a bending cantilever beam (BCB) [6]. In this paper, we propose and demonstrate a novel liquid level sensor based on the measurement of the short-wavelength-loss peaks of FBGs. The proposed scheme has the following advantages- (1) they are robust (etched FBGs are fragile); (2) they are simple (no need to design BCB); (3) their sensitivity can be flexibly and widely chosen since potentially tens of peaks can be used in one FBG (compared to LPGs which just have a few peaks can be used); (4) temperature can be simultaneously and independently measured from its Bragg resonance (the schemes based on LPG and etched FBG need extra elements to measure temperature).

\section{Experiment}

The level test setup (shown in Fig.1a) consisted of a 50mm-long uniform FBG, a plastic tube (serving as liquid container), supporting holders, a broadband light source and an optical spectrum analyzer. The plastic tube initially had a small hole at the bottom, which allowed the optical fiber to go through and was then tightly sealed to avoid liquid leak. The FBG position was adjusted to be appropriate in the tube so that the whole length can be tested. The optical fiber was mounted vertically with a tiny strain applied to avoid possible bending effect. The liquid level was adjusted by injecting liquid into the tube. A liquid with refractive index of $\sim 1.44$ was used in initial tests. Before the liquid level reached the start point of the FBG, the monitored transmission spectrum had no change. The measured transmission spectrum of the 
FBG in air is shown in Fig.1b. It is seen in Fig.1b that the original FBG had lots of distinct loss peaks in the short wavelength range, which were due to the coupling of light from the core mode to symmetric cladding modes $\mathrm{LP}_{0, \mathrm{~m}}$ $(\mathrm{m}=2,3, \ldots)$. When the liquid level reached the FBG, some interesting changes were observed in the transmission spectrum, as seen in Fig.2a. For lower order modes $(\mathrm{m}<=6)$, the spectral profile were hardly changed, which means lower order modes are much less sensitive to surrounding refractive index change. For each higher order modes $(\mathrm{m}>6)$, an additional new peak adjacent to the original mode appeared at its long wavelength side. The corresponding new peak was due to coupling to the same asymmetric cladding modes from the immersed part of the FBG. The higher mode order, the wider the separation of the two peaks, which indicated the sensitivity increased with the mode order. For those peaks with a separation wide enough, one can see clearly that the new peaks grew stronger as the immersion length increasing, while the original peaks became weaker at same time. Fig.2c shows the amplitudes of the original $\mathrm{LP}_{0,12}$ peak and its corresponding new peak as a function of immersion length. The amplitude difference of the two peaks is also plotted in Fig.2c, which has near linear response and can be a good candidate for level measurement. It is also interesting to note

that the Bragg resonance is not sensitive to the surrounding refractive index at all, which means it can act as an independent temperature indicator in simultaneous measurements. The temperature response of the FBG was measured in an environmental chamber, in which the temperature was varied from $10^{\circ} \mathrm{C}$ to $60^{\circ} \mathrm{C}$. The wavelengths of the Bragg resonance and the $\mathrm{LP}_{0,12}$ peak as a function of temperature is plotted in Fig.3a, in which both can be seen to have a good linear response to temperature. The level sensing test was also carried out in air conditioned room with room temperature setting of $15^{\circ} \mathrm{C}$ and $30^{\circ} \mathrm{C}$ and the results were shown in Fig. $3 \mathrm{~b}$. It is seen in Fig. $3 \mathrm{~b}$ that the results for two temperatures are very close.

We further tested a liquid with refractive index close to refractive index of optical fiber $(\sim 1.45)$ and the results were shown in Fig.4. There are some different features in the spectral profile with increasing liquid level, as seen in Fig.4a. Unlike in Fig.2a, no new peaks appeared near the original peaks and the transmission loss (radiation modes induced loss) increases with increasing immersion length. The amplitude of the original cladding resonance peaks decrease with increasing immersion length and the peaks almost disappear when the full length of the FBG is immersed. This level test was also conducted in room with temperature setting of $15^{\circ} \mathrm{C}$ and $30^{\circ} \mathrm{C}$ and the results were shown in Fig $4 \mathrm{~b}$ and $4 \mathrm{c}$. Again the results are very close for the two temperatures.

\section{Conclusion}

We propose and demonstrate a novel liquid level sensor based on the measurement of the short-wavelength-loss peaks of FBGs. Such a scheme has some advantages including robustness, simplicity, flexibility in choosing sensitivity, and simultaneous temperature measurement capability.

Acknowledgement: The authors acknowledge the support of the UK EPSRC for this work.

\section{References}

[1] I. K. Llev and R. W. Waynant, “All-fiber-optic sensor for liquid level measurement,” Rev. Sci. Instr. 70, 2551-2554 (1999).

[2] E. Musayev and S. E. Karlik, "A novel liquid level detection method and its implementation," Sens. Actuators A, 109, 21-34 (2003).

[3] K. E. Romo-Medrano and S. N. Khotiaintsev, "An optical-fibre refractometric liquid-level sensor for liquid nitrogen.” Mea. Sci. Technol. 17, 998-1004 (2006).

[4] S. Khaliq, S. W. James, and R. P. Tatam, "Fiber-opitc liquid-level sensor using a long-peirod grating," Opt. Lett., 26, 1224-1226, (2001). 
[5] T. Guo, Q. Zhao, Q. Dou, H. Zhang, L. Xue, G. Huang, and X. Dong, "Temperature-insensitive fiber Bragg grating liquid-level sensor based on bending cantilever beam," IEEE Photon. Technol. Lett., 17, 2400-2402, (2005).

[6] B. Yun, N. Chen, and Y. Cui, "Highly Sensitive Liquid-Level Sensor Based on Etched Fiber Bragg Grating," PTL, 19, 1747-1749, (2007).

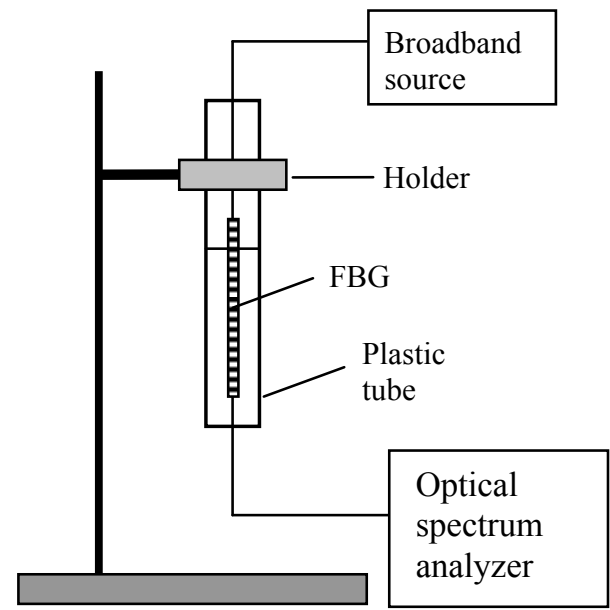

(a)

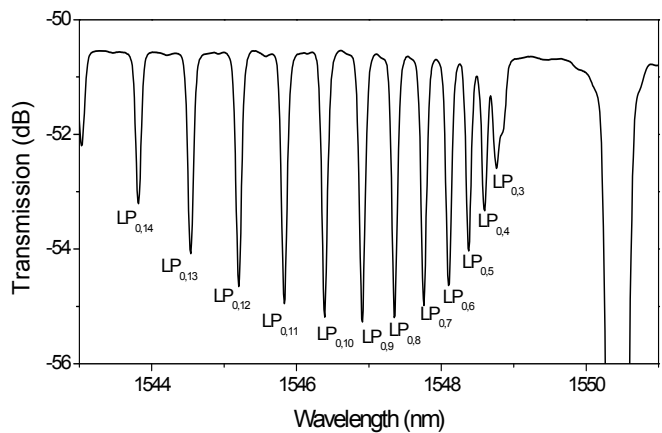

(b)

Fig.1 (a) Schematic of the liquid-level measurement setup. (b) Transmission spectrum of FBG in the air.

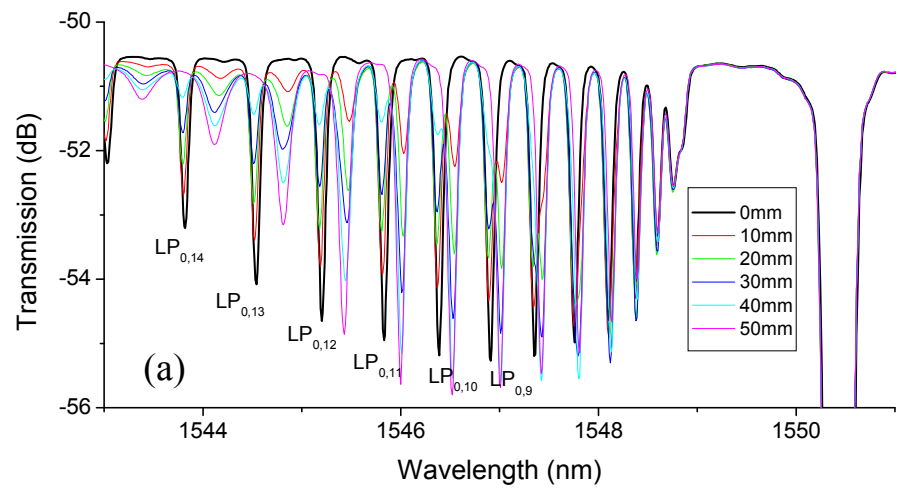

(b)
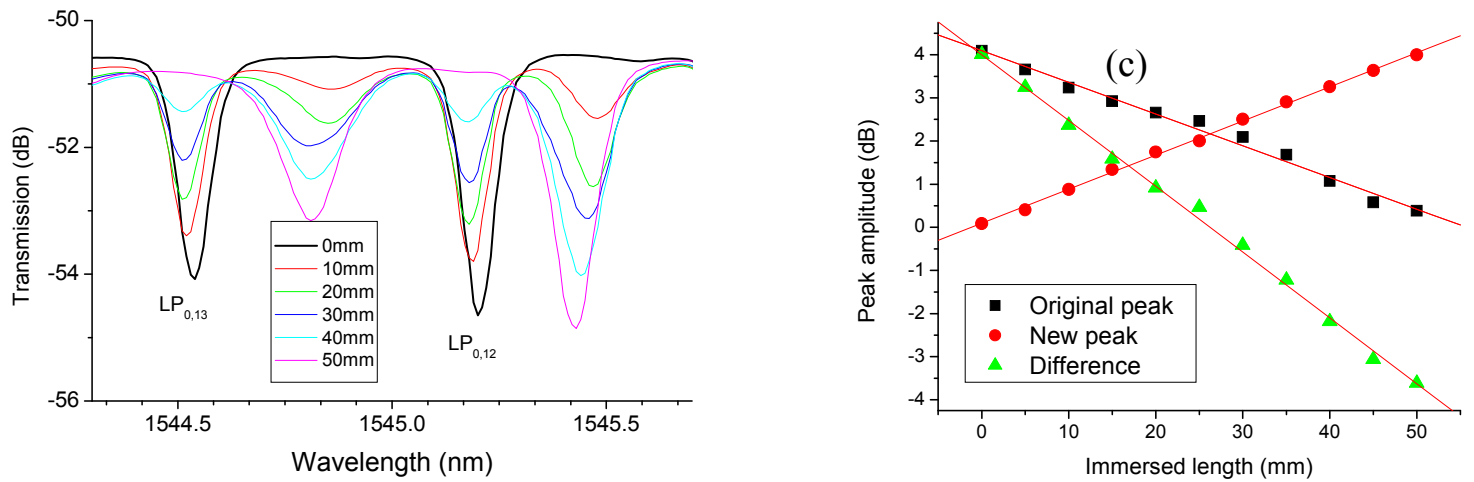

Fig.2 (a) Transmission spectra of the FBG at different immersion lengths. (b) Enlarged part of (a). (c) $\mathrm{LP}_{0,12}$ mode resonance as a function of immersion length. 

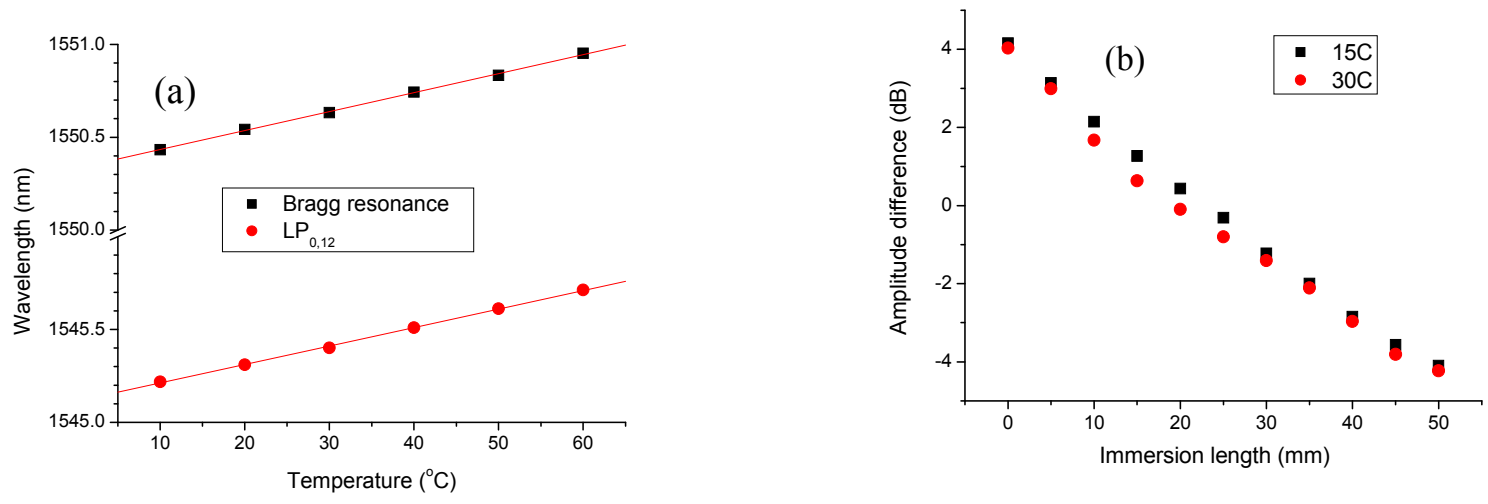

Fig.3. (a) Bragg resonance and $\mathrm{LP}_{0,12}$ mode resonance as a function of temperature. (b) Amplitude difference of $\mathrm{LP}_{0,12}$ mode resonance as a function of immersion length at $15^{\circ} \mathrm{C}$ and $30^{\circ} \mathrm{C}$.
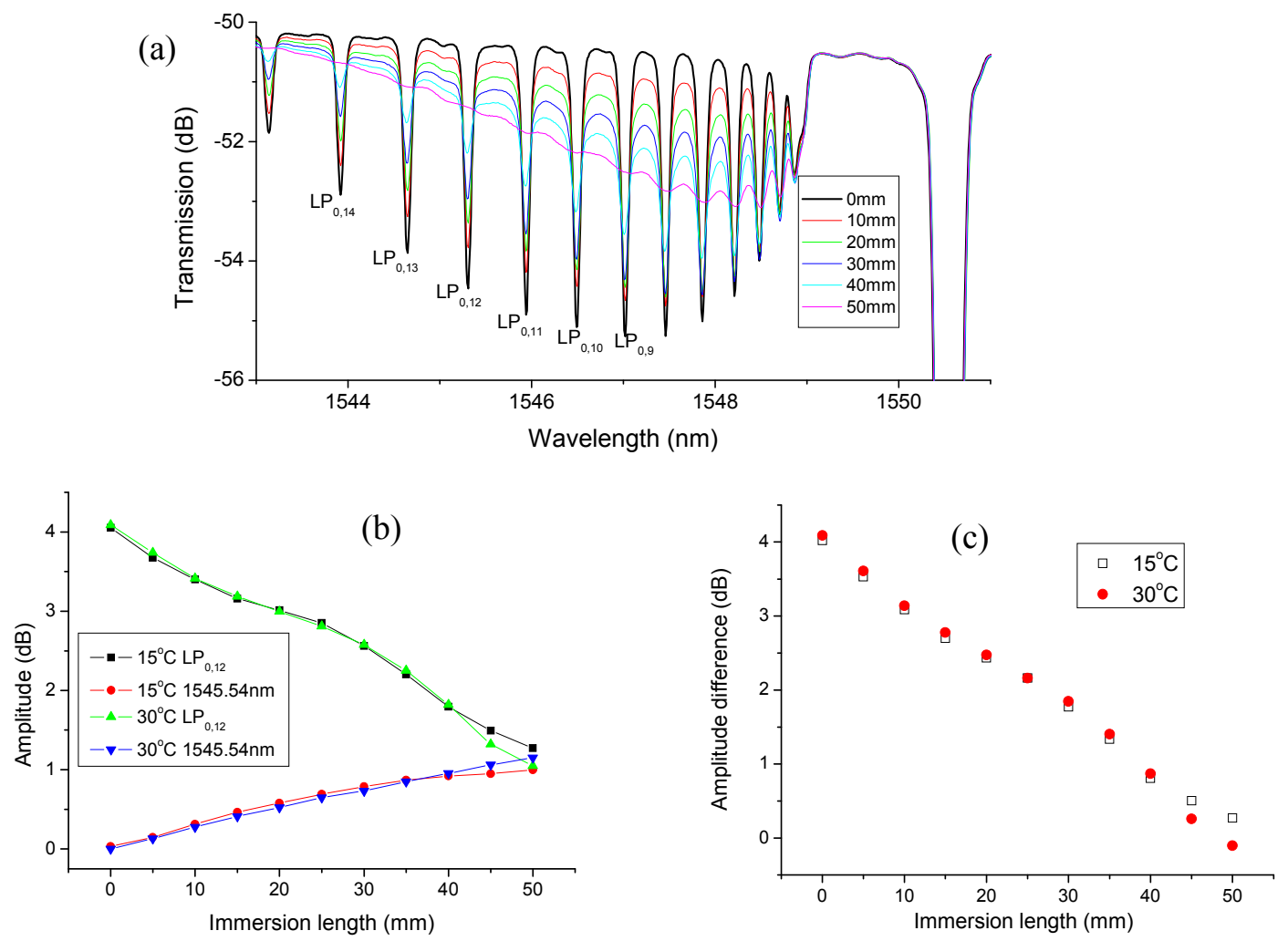

Fig.4 (a) Transmission spectra of an FBG at different immersion lengths in liquid with refractive index close to the cladding index. (b) $\mathrm{LP}_{0,12}$ mode resonance and loss at $1545.54 \mathrm{~nm}$ as a function of immersion length at $15^{\circ} \mathrm{C}$ and $30^{\circ} \mathrm{C}$. (c) Amplitude difference of $\mathrm{LP}_{0,12}$ mode resonance as a function of immersion length at $15^{\circ} \mathrm{C}$ and $30^{\circ} \mathrm{C}$. 